\title{
Benchmarking ensemble docking methods as a scientific outreach project
}

\author{
${ }_{3}$ Jessie L. Gan ${ }^{1}$, Dhruv Kumar ${ }^{2}$, Cynthia Chen ${ }^{3,6}$, Bryn C. Taylor ${ }^{4,7}$, Benjamin R. Jagger ${ }^{4,8}$, Rommie E. Amaro ${ }^{4,}$, and
} ${ }_{4}$ Christopher T. Lee ${ }^{5, *}$

${ }_{5}^{1}$ San Diego Jewish Academy, San Diego, CA 92130

${ }_{6}^{2}$ Rancho Bernardo High School, San Diego, CA 92128

$7^{3}$ Canyon Crest Academy, San Diego, CA 92130

${ }_{8}^{4}$ Department of Chemistry and Biochemistry, University of California San Diego, La Jolla, CA 92093

$9^{5}$ Department of Mechanical and Aerospace Engineering, University of California San Diego, La Jolla, CA 92093

${ }_{10}^{6}$ Current Address: California Institute of Technology, Pasadena, CA 91125

${ }_{11}^{7}$ Current Address: Discovery Sciences, Janssen Research and Development, San Diego, CA 92121

${ }_{12}^{8}$ Current Address: Department of Bioengineering and Therapeutic Sciences, University of California San Francisco, San

${ }_{13}$ Francisco, CA 94158

${ }_{14}$ *Correspondence: ramaro@ucsd.edu, ctlee@ucsd.edu

${ }_{15}$ ABSTRACT The discovery of new drugs is a time consuming and expensive process. Methods such as virtual screening, which ${ }_{16}$ can filter out ineffective compounds from drug libraries prior to expensive experimental study, have become popular research 17 topics. As the computational drug discovery community has grown, in order to benchmark the various advances in methodology, ${ }_{18}$ organizations such as the Drug Design Data Resource have begun hosting blinded grand challenges seeking to identify the ${ }_{19}$ best methods for ligand pose-prediction, ligand affinity ranking, and free energy calculations. Such open challenges offer a ${ }_{20}$ unique opportunity for researchers to partner with junior students (e.g., high school and undergraduate) to validate basic yet ${ }_{21}$ fundamental hypotheses considered to be uninteresting to domain experts. Here, we, a group of high school-aged students and 22 their mentors, present the results of our participation in Grand Challenge 4 where we predicted ligand affinity rankings for the ${ }_{23}$ Cathepsin S protease, an important protein target for autoimmune diseases. To investigate the effect of incorporating receptor ${ }_{24}$ dynamics on ligand affinity rankings, we employed the Relaxed Complex Scheme, a molecular docking method paired with 25 molecular dynamics-generated receptor conformations. We found that CatS is a difficult target for molecular docking and we ${ }_{26}$ explore some advanced methods such as distance-restrained docking to try to improve the correlation with experiments. This ${ }_{27}$ project has exemplified the capabilities of high school students when supported with a rigorous curriculum, and demonstrates 28 the value of community-driven competitions for beginners in computational drug discovery.

Keywords: Computational Biophysics; Ensemble Docking; Molecular Dynamics; Drug Discovery; Secondary Education; ${ }_{29}$ Undergraduate Education; Outreach Coordinators

30

\section{${ }_{31} 1$ INTRODUCTION}

${ }_{32}$ Drug discovery efforts often require the screening of many зз compounds to determine their efficacy. Owing to the high cost ${ }_{34}$ of experimental screening and advances in computer models, ${ }_{35}$ the use of inexpensive computational screening methods to ${ }_{36}$ enrich compounds in large datasets have been used in drug ${ }_{37}$ discovery pipelines for several decades (1). Early on in the 38 screening process, when an initial compound library may 39 contain only a few 'active' among many orders of magnitude 40 more inactive compounds, computer-aided drug discovery ${ }_{41}$ (CADD) methods, such as virtual screening, can be used to 42 filter out unlikely candidates, reducing experimental costs, ${ }_{43}$ and accelerating the initial discovery phase (2-4).

${ }_{44}$ Due to the diversity and breadth of the CADD research ${ }_{45}$ community, many methods have been developed. Cross${ }_{46}$ comparison and benchmarking between the different ap47 proaches is necessary for identifying the limitations of the 48 docking method and areas for improvement. The Drug Design ${ }_{49}$ Data Resource (D3R) hosts blinded community prediction
${ }_{50}$ challenges to evaluate these software and techniques and com${ }_{51}$ pare their effectiveness on benchmark systems, such as the ${ }_{52}$ HSP90 chaperone protein, the Farnesoid X nuclear receptor, ${ }_{53}$ and the Cathepsin S protease (CatS) (5-7). In 2018, D3R ${ }_{54}$ hosted Grand Challenge 4 (GC4), which had components of ${ }_{55}$ pose prediction, free energy prediction, and ligand affinity 56 rank ordering (8).

We participated in Subchallenge 2, a ligand affinity ranking 58 challenge for the Cathepsin $\mathrm{S}$ protease with a set of 459 ligands ${ }_{59}$ provided by Janssen Pharmaceuticals (8). CatS is a cysteine 60 protease involved in the presentation of antigens by the MHC ${ }_{61}$ class II molecules within $\mathrm{CD}^{+}{ }^{+} \mathrm{T}$ cells (9). This makes it a 62 promising target in autoimmune disease and allergy treatment, 63 where inhibition of the immune response is critical for effective 64 therapy (10-12).

We used molecular docking, a popular method of vir${ }_{66}$ tual screening, in a strategy known as the Relaxed Complex ${ }_{67}$ Scheme to account for protein flexibility (13). Molecular 68 docking applies a conformational search algorithm paired 
${ }_{69}$ with an inexpensive, and often empirical, scoring function to 70 find favorable lead compounds $(14,15)$. By forgoing rigorous ${ }_{71}$ dynamics and detailed potential energy functions, such as 72 those used in free energy calculations, docking approaches ${ }_{73}$ are designed to yield results quickly albeit with lower accu${ }_{74}$ racy (16). The speed of molecular docking codes enables the 75 screening of hundreds of thousands to millions of compounds 76 (17). A risk of docking is the increased likelihood of false 77 negatives. To this end, much work has been done by the 78 community to develop improved algorithms which improve 79 docking accuracy with minimal impact on speed (3).

so In early docking studies, proteins and ligands were repre${ }_{81}$ sented as static structures $(16,18,19)$. To incorporate ligand ${ }_{82}$ flexibility, multiple ligand positions can be sampled through 8s rotational torsions, i.e. conformer generation (20). However, ${ }_{84}$ Molecular Dynamics (MD) simulations have revealed that 85 thermal protein fluctuations in solute-based environments ${ }_{86}$ can give rise to varying conformational states, resulting in ${ }_{87}$ different binding sites (21). Accounting for receptor binding ${ }_{88}$ site flexibility in molecular docking is a significant challenge.

${ }_{89}$ One solution is to perform ensemble docking. This involves

90 docking a ligand compound library to a number of distinct,

${ }_{91}$ rigid receptor conformations to identify the receptor confor-

92 mation that is best suited for that particular ligand (i.e. best

${ }_{93}$ docking score) (20, 22-24).

${ }_{94}$ Here, we perform MD simulations of the receptor protein, ${ }_{95} \mathrm{CatS}$, to obtain unique conformational states and introduce 96 structural variation in the binding site. MD simulations allow ${ }_{97}$ the exploration of multiple conformations of the protein while ${ }_{98}$ in a solute-based, native environment $(25,26)$. This concept of 99 selecting naturally-occurring conformations through MD for 100 ensemble docking is known as the Relaxed Complex Scheme 101 (26-31). MD-generated ensembles of flexible binding sites 102 have been used successfully in a number of studies to identify 103 lead compounds (13, 32-35).

104 Incorporating more receptor conformations increases com${ }_{105}$ putational cost, as a complete docking protocol must be per106 formed for each conformation. To address this, the trajectory ${ }_{107}$ can be clustered to extract unique, representative conforma108 tions $(36,37)$. This methodology is still susceptible to the 109 conformational sampling problem of MD, due to the large ${ }_{110}$ discrepancy between the accessible timescales of MD simula111 tion (microseconds) and the slow, native dynamics of proteins 112 (milliseconds and longer) $(38,39)$. Although a trajectory may 113 not statistically converge to encompass all possible conforma114 tions, studies have shown that clustering MD trajectories can 115 reveal previously unknown druggable pockets (32).

116 Many studies have successfully used clustering methods 117 in ensemble docking to extract relevant conformations, such 118 as those based on $\operatorname{RMSD}(26,34)$, QR factorization $(13,33)$, ${ }_{119}$ and active pocket volume (26). However, choosing the most ${ }_{120}$ appropriate clustering method for a system is still challenging ${ }_{121}$ and often dependent on human intuition.

122 Although ensemble docking has been successfully used ${ }_{123}$ to identify lead compounds, clustering methods in ensemble
124 docking have not been extensively studied (40). We explored 25 three clustering methodologies in this study to investigate ${ }_{126}$ if they could (i) provide an accurate ligand ranking and (ii) ${ }_{127}$ give insights into CatS ligand binding mechanisms. The three ${ }_{128}$ clustering methods we used are: 1) Time-lagged Independent ${ }_{129}$ Components Analysis and K-means clustering (TICA) (41, 130 42), 2) Principal Component Analysis and K-means clustering ${ }_{131}$ (PCA) (41, 42), and 3) Gromos RMSD clustering (Gromos) ${ }_{132}$ (43). TICA identifies the slowest motions of the simulation ${ }_{133}$ and projects the input features into a slow subspace where ${ }_{134}$ distinct clusters are kinetically separated (44). PCA, on the ${ }_{35}$ other hand, finds features with the largest variance (45). Lastly, ${ }_{36}$ Gromos is a RMSD-based clustering method that counts the ${ }_{37}$ neighbors in a cluster based on a pre-set cutoff value and 138 defines trajectory clusters by structural variation (43).

${ }_{139}$ In this work, we apply the Relaxed Complex Scheme 140 with these clustering methods and compare the ensemble ${ }_{141}$ docking results (30). We test the accuracy of two state-of-the${ }_{142}$ art docking softwares: Open Eye FRED (46) and Schrodinger ${ }_{143}$ Glide (47). We found that CatS is a difficult target for molecular ${ }_{144}$ docking and we explore some advanced methods such as 145 distance-restrained docking to try to improve the correlation 146 with experiments.

\section{PEDAGOGICAL SIGNIFICANCE}

${ }_{148}$ This manuscript presents the work of high school students 149 who have performed this work after completing BioChem${ }_{150} \mathrm{CoRe}$, a 7 week crash course on computational chemistry 151 (http://biochemcore. ucsd. edu/). These results helps to 152 illustrate the benefits and possibilities of teaching science ${ }_{153}$ as we do science $(48,49)$. By participating in structured ${ }_{154}$ challenges with real-world significance, students gain motiva155 tion, confidence, and both technical and soft skills. Moreover, 56 the exposure to the rigors of the scientific approach and the methods employed in the field of study aids them with their ${ }_{158}$ future career decisions. On the other hand, community-driven 59 competitions and resources such as D3R's Grand Challenge 4 iso can also benefit from student participation. Rarely do these ${ }_{61}$ programs receive submission which test the basic hypothesis. ${ }_{162}$ For example, is domain expertise required for the application ${ }_{163}$ of the methods of interest? Given the current state of tutorials ${ }_{164}$ or instructions available to the public, can students with lim165 ited domain experience use these resources to produce results 166 without major technical difficulties? We posit that student ${ }_{167}$ participation can not only yield important benchmarking data ${ }_{168}$ but also serve to improve the documentation of our tools and 69 methods.

\section{${ }_{170} 3$ MATERIALS AND METHODS}

${ }_{171}$ All scripts used in this work can be found online at https: $172 / /$ github.com/ctlee/bccgc4. Full workflow of methods ${ }_{173}$ is shown in Fig. 1. 


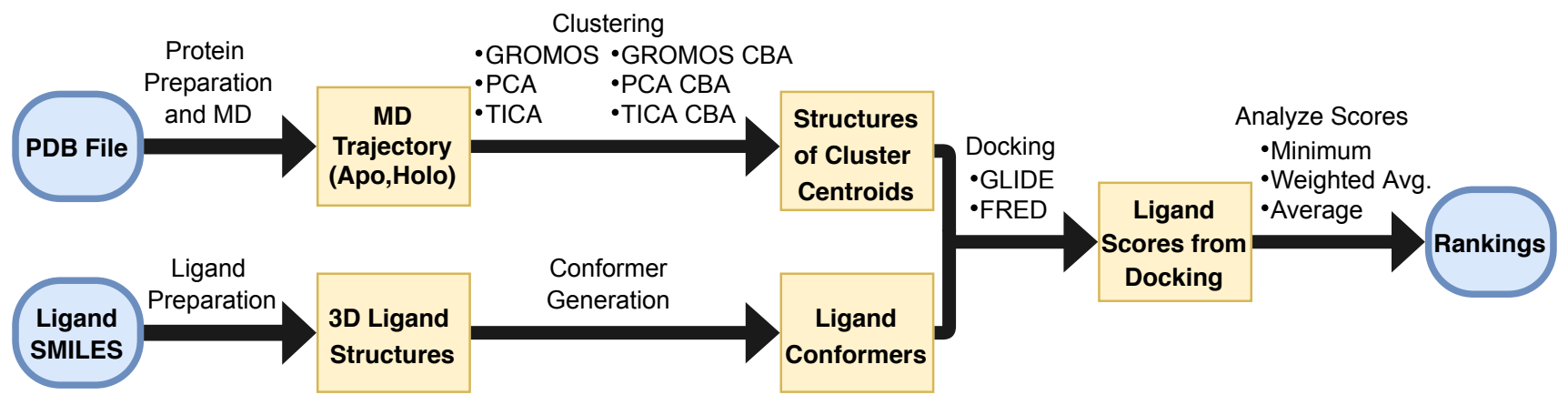

Figure 1: Workflow of ensemble docking approach. PDB file was selected and simulated in Molecular Dynamics. Molecular Dynamics trajectory was clustered by six various methods and cluster centroids were extracted as representative structures. Ligand SMILES were prepared as 3D structures and various conformers were generated. Molecular docking of ligands to cluster centroids was performed with FRED and Glide docking. Pose scores were used to generate rank orderings and Kendall's $\tau$ values when compared to the experimental rank ordering.

\section{3.1 Molecular Dynamics}

175 A crystal structure of CatS (PDBID: 5QC4 (11)) was obtained 176 from the RCSB PDB database (50). The structure was chosen 177 due to its resolution of $2 \AA$ and similarity of the cocrystallized 178 ligand to those in the D3R dataset. This cocrystal was part of ${ }_{179}$ D3R's prior Grand Challenge 3 (GC3), subchallenge 1, and 180 the ligands in the CatS subchallenge of Grand Challenge 4 all ${ }_{181}$ contain the tetrahydropyrido-pyrazole core that 22 of the 24 182 ligands had in the previous challenge (7).

183

Models with (holo) and without (apo) the cocrystallized 184 ligand were prepared for MD simulations. For both apo 185 and holo models the same steps were performed with a few 186 deviations noted below. Chain A of the structure was prepared 187 in Schrodinger Maestro 2019 (51) with the Protein Preparation ${ }_{188}$ Wizard. For the holo simulation, the cocrystallized ligand was 189 retained. For the apo simulation the ligand was removed (52). 190 Force field parameters for the ligand were derived from GAFF ${ }_{191}$ (53) with partial charges fit using the restrained electrostatic 192 potential method (RESP) (54) from potentials computed 193 using the AM1-BCC semi-empirical quantum mechanical 194 method $(55,56)$. For both systems, the protein termini were 195 capped with an acetyl (ACE) and N-methyl amide (NME) 196 capping groups. PROPKA $(57,58)$ was used to assign residue 197 protonation states in a solvent of $\mathrm{pH} 5.0$, to mimic experimental ${ }_{198}$ conditions of CatS binding assays (9). Crystal waters with 199 more than 2 hydrogen bonds to non-waters were retained.

${ }_{200}$ Using a combination of pdb4amber and tleap from the ${ }_{201}$ AMBER 18 software suite, we parameterized the systems 202 with the AMBER FF14SB forcefield, and solvated the systems ${ }_{203}$ with TIP4P-Ew up to a $15 \AA$ A buffer distance (59). We added 204 ions according to the SLTCAP tool by Schmit et al. (60) 205 at $100 \mathrm{mM}$ salt concentration, again to mimic experimental 206 conditions (9).

${ }_{207}$ All-atom, explicit-solvent MD simulation was performed ${ }_{242}$ To identify a good set of initial input features, we compared ${ }_{208}$ for the both systems using AMBER18 in four stages: min- ${ }_{243}$ the mean 10-fold cross-validated Variational Approach for ${ }_{209}$ imization, heating, equilibration, and production (59). The ${ }_{244}$ Markov Processes (VAMP2) scores for three selections: i) ${ }_{210}$ systems were gradually minimized in four steps: (i) mini- 245 protein backbone atom positions, ii) protein backbone tor-
211 mization of only protons, restraining the protein and solvent, 212 (ii) minimization of the solvent, restraining the protein, (iii) 213 minimization of the protein sidechains, restraining the protein 214 backbone, and (iv) minimization of all atoms. Restrained heat215 ing was performed in two steps: first, in the NVT ensemble ${ }_{216}$ the temperature was increased from 0 to $100 \mathrm{~K}$ over $50 \mathrm{ps}$ ${ }_{217}$ using a Langevin thermostat, and second in the NPT ensemble ${ }_{218}$ the temperature was increased from 100 to $300 \mathrm{~K}$ over $200 \mathrm{ps}$ ${ }_{219}$ using a Langevin thermostat while pressure was maintained 220 at 1 bar using a Berendesen barostat. Equilibration was also ${ }_{221}$ performed in two stages, first with a restrained backbone, 222 and second without restraints. For both equilibration stages ${ }_{223}$ the temperature was maintained at $300 \mathrm{~K}$ using a Langevin 224 thermostat. For the restrained equilibration stage, 500 ps were ${ }_{225}$ run with a Berendsen barostat to equilibrate pressure to $1 \mathrm{bar}$. ${ }_{226}$ In the unrestrained equilibration step 1000 ps were run using ${ }_{227}$ a Monte Carlo barostat at 1 bar. 229 with the same conditions as the unrestrained equilibration 230 step. Five independent simulations of each condition, apo and ${ }_{231}$ holo, of length $2 \mu \mathrm{s}$ were run, totaling $20 \mu \mathrm{s}$. Hydrogen Mass ${ }_{232}$ Repartitioning (HMR) was performed with PARMED (59, ${ }_{233} 61$ ) for all systems permitting a 4 fs timestep. All simulations ${ }_{234}$ were run with SHAKE restraints (62) and a non-bonded cutoff 235 of $10 \AA$.

\section{${ }_{236} 3.2$ Clustering}

${ }_{237}$ The MD trajectory was clustered using three different clus${ }_{238}$ tering methods: 1$)$ TICA and k-means $(41,42,63,64)$ on 239 the protein backbone atom position coordinates, 2) PCA and $240 \mathrm{k}$-means on the protein backbone atom position coordinates, ${ }_{241}$ and 3) Gromos (43) on the C-alpha atom position coordinates.
Production simulations were run in the NPT ensemble 
246 sions, and iii) the positions of a binding atoms selection (65). 2 ${ }_{247}$ We decided to use the positions of protein backbone atoms ${ }_{248}$ because it had the largest VAMP2 score, indicating greater ${ }_{249}$ kinetic variance. The binding atoms were defined by taking ${ }_{250}$ all receptor atoms within $2 \AA$ of the initial docked poses of a ${ }_{251}$ ligand from the D3R data-set. PCA was clustered on the same ${ }_{252}$ subset of backbone atom positions (41), and Gromos was ${ }_{253}$ clustered on the $\mathrm{C}$-alpha positions due to memory limitations. ${ }_{254}$ After the challenge, the clustering was reevaluated and a sec255 ond discretization using the binding atoms selection, referred 256 to as Clustered by Binding Atoms (CBA), was generated. We ${ }_{257}$ used similar ideas as the approach taken in Ref. (66), focusing 258 on the binding site's structural fluctuations rather than the 259 entire structure. All six clustering methods (TICA, PCA, and ${ }_{260}$ Gromos for backbone or C-alpha atoms and CBA) were also ${ }_{261}$ performed on the holo MD trajectories. The cluster centroids ${ }_{262}$ of the apo MD were compared by pairwise RMSD, utilizing ${ }_{263}$ MDTraj and NumPy together to calculate RMSDs and order 264 them into a matrix which was visualized in matplotlib (67-69). 265 They were also compared in terms of Root-Mean-Squared${ }_{266}$ Fluctuation (RMSF) to investigate the particular structural ${ }_{267}$ variability, computed in MDTraj and visualized in PyMOL $268(68,70)$.

\section{${ }_{269}$ 3.2.1 Time-lagged Independent Components Analysis ${ }_{270} \quad$ and K-means (TICA)}

${ }_{271}$ TICA clustering was employed to capture the slow motions 272 within the trajectory. TICA was performed with a lag time of ${ }_{273} 4 \mathrm{ps}$ and a variance cutoff of 0.95 on the protein backbone ${ }_{274}$ atom coordinates (41). The trajectory was projected into 275 the TIC basis and subsequently, the k-means algorithm was ${ }_{276}$ used to cluster the trajectory into 10 distinct clusters. The 10 ${ }_{277}$ configurations from the trajectory, in real space, closest in ${ }_{278}$ TIC space to the cluster centroids were used for docking (42).

\section{3.2.2 Principal Components Analysis and K-means $280 \quad$ (PCA)}

${ }_{281}$ PCA with a variance cutoff of 0.95 was performed on the ${ }_{282}$ protein backbone atom coordinates to capture large motions ${ }_{283}$ within the trajectory (41). The trajectory was projected into the ${ }_{284} \mathrm{PC}$ basis and subsequently, the k-means algorithm was used 285 to cluster the trajectory into 10 clusters. The 10 configurations ${ }_{286}$ from the trajectory, in real space, closest in PC space to the ${ }_{287}$ cluster centroids were used for docking (42).

\section{3.2.3 Gromacs RMSD-Based Clustering (Gromos)}

${ }_{289}$ Gromos clustering was performed on the alpha carbons in 290 the protein to identify structurally diverse conformations ${ }_{291}$ according to RMSD (43). The trajectories input to Gromos 292 were subsampled to yield frames every $0.4 \mathrm{ps}$. This was due to ${ }_{293}$ computational intractability at more frequent frame rates. The ${ }_{294}$ clustering RMSD cutoff was chosen to satisfy the following 295 criteria: (i) the first cluster had less than $70 \%$ of the frames,
296 (ii) the first 10 clusters contained at least $80 \%$ of the frames, 297 and (iii) each of the first 10 clusters had at least 20 frames. A 298 cutoff of $0.08 \AA$ was used when clustering with alpha carbons 299 while a cutoff of $0.15 \AA$ was used when compared to CBA 300 for the apo trajectory. A cutoff of $0.07 \AA$ was used when 301 clustering with alpha carbons while a cutoff of $0.135 \AA$ was 302 used when compared to CBA for the holo trajectory.

\section{${ }_{303}^{3.3}$ Docking}

${ }_{304}$ OpenEye Scientifics Fast Exhaustive Docking (FRED) was 305 used to dock the 459 ligands in the GC4 CatS challenge to 306 the centroids of the clustered MD trajectory and the original 307 crystal structure (46). After the challenge, Schrodingers Glide 308 was also used in an attempt to improve rank ordering and so9 pose prediction $(47,71)$. In addition, many iterations of Glide 310 docking were run with modifications to further improve the ${ }_{311}$ results. The pose results were visualized in Schrodinger's ${ }_{312}$ Maestro (51) and labeled in Inkscape (72). The pose results 313 were analyzed for accuracy through the RMSD of the common ${ }_{314}$ core to the original cocrystal ligand core, calculated using ${ }_{315}$ Schrodinger's Python API and visualized in matplotlib (69).

\section{${ }_{316}$ 3.3.1 FRED}

${ }_{317}$ OpenEye's OMEGA was used to convert ligand SMILES to ${ }_{318}$ 3D conformers, with the maximum number of conformers ${ }_{319}$ per ligand set to 800 (73). The conformers were then docked ${ }_{320}$ to the crystal structure and the 10 cluster centroids from ${ }_{321}$ each clustering method using OEDockings FRED default ${ }_{322}$ settings (Chemgauss4 scoring function with standard search ${ }_{323}$ resolution) $(46,74)$. The receptor area was defined by a box 324 around the protein, determined by the minimum and maximum ${ }_{325}$ distance coordinates of the entire protein. For each receptor ${ }_{326}$ ensemble, the minimum score of every ligand was used in 327 determining the rank ordering, as in previous studies $(13,32)$.

\section{3.3.2 Glide}

${ }_{329}$ Schrodingers Ligprep was used to convert ligand SMILES us${ }_{3 з 0}$ ing standard settings into Maestro structures for Schrodingers ${ }_{331}$ Glide docking (75). Glides cross-docking script, xglide.py, ${ }_{332}$ was used to perform ensemble docking for each clustering ${ }_{3 з 3}$ method. The cross-docking script generated receptor grid files ${ }_{334}$ for each centroid structure using a $32 \AA$ box centered on the 335 center of mass of the crystal structure's ligand (BC7 (11)) to 336 define the docking region. Each centroid was then docked to ${ }_{337}$ using Glides Standard Precision (SP) docking methodology, ${ }_{338}$ which has its own ligand conformer generation steps, and 339 scored with the subsequent Standard Precision GlideScore 340 scoring function $(47,71)$. For each ensemble docking ap${ }_{341}$ proach, the best score of each ligand across the ensemble of 342 conformations (N) was used to determine its rank,

$$
s_{l}=\min \left\{s_{l, i}: i \in \mathbf{N}\right\},
$$


${ }_{343}$ where $s_{l}$ and $s_{l, i}$ are the best overall score and best score for 344 receptor conformation $i$ for ligand $l$ respectively.

${ }_{345}$ To further investigate the ligand binding we also 1) applied 346 a restraint on the tetrahydropyrido-pyrazole common core ${ }_{347}$ structure, restricted to lie within $3.5 \AA$ of the cocrystal ligand's ${ }_{348}$ common core, 2) changed the precision of the docking and 349 scoring function from Glide SP to Glide Extra Precision (XP) ${ }_{350}$ (76), and 3) clustering and docking to centroids from a holo ${ }_{351}$ MD trajectory.

\section{${ }_{352} 3.4$ Scoring Schemes for Ligand Scores}

${ }_{353}$ Aside from changing the docking methodology, we also tried ${ }_{354}$ two other scoring schemes such as taking the average and the ${ }_{355}$ weighted average (Eq. (2)) of the OpenEye's FRED and Glide ${ }_{356}$ SP scores.

$$
s_{l}=\sum_{i \in \mathbf{N}} P_{i} * s_{l, i} .
$$

${ }_{357}$ where $P_{i}$ is the probability of observing conformation $i, s_{l, i}$ is ${ }_{358}$ the best docked score for that conformation, and $\mathbf{N}$ is the set 359 of conformations in the ensemble. Note that the probabilities, ${ }_{360} P_{i}$, are normalized such that $\sum_{i \in \mathbf{N}} P_{i}=1$. The $P_{i}$ for a given ${ }_{361}$ conformation $i$ is calculated as $f_{i} / f_{T}$, where $f_{i}$ is the number 362 of frames in the same cluster as $i$, and $f_{T}$ is the total number ${ }_{363}$ of frames in the trajectory. These scoring schemes have been ${ }_{364}$ used in other studies due to the reasoning that the average (33, $\left.{ }_{365} 77\right)$ or weighted average $(34,35)$ score better accounts for the ${ }_{366}$ variability of the ensemble, and in the case of the weighted 367 average, represents the likelihood of the ligand encountering ${ }_{368}$ each representative conformation in a natural environment.

\section{${ }_{369}$ 3.5 Kendall's Taus}

${ }_{370}$ Ligand rankings were created by sorting the ligands based on ${ }_{371}$ their score. Kendall's Tau values were calculated by comparing 372 the predicted rank ordering to the experimental rank ordering 373 using the Kendall's Tau function in SciPy (78).

\section{${ }_{374} 4$ RESULTS AND DISCUSSION}

${ }_{375}$ In lieu of running expensive free energy calculations which ${ }_{376}$ account for both ligand and receptor flexibility, the Relaxed ${ }_{377}$ Complex Scheme attempts to reduce computational cost while ${ }_{378}$ capturing the flexibility of a protein by docking to multiple 379 protein conformations selected from a MD simulation. These sво representative conformations are often chosen by combining ${ }_{381}$ a method of dimensionality reduction followed by the appli382 cation of a clustering algorithm. Although this approach is 383 conceptually simple, the choice of clusters has many pitfalls. ${ }_{384}$ For example, even if a set of clusters spans the conforma385 tional diversity of the MD trajectory, the ensemble will not ${ }_{386}$ necessarily produce the most accurate ligand rank ordering ${ }_{387}$ (40). Some receptors may have natural conformations which ${ }_{388}$ are not ideal for ligand binding, and these may result in false s89 positives (25). In addition, the active conformation for ligand
390 binding could be transient, and would have a lower probability 391 of being represented in the ensemble.

392 To test the effect of clustering approach on the resulting ${ }_{393}$ conformations, we test several different clustering methods. 394 TICA captures slow protein movements (variance in time), 395 while PCA focuses on large structural variance, and Gromos 396 captures structural variations as measured by RMSD. We ${ }_{397}$ plot the structural variation across clusters from the different 398 algorithms in Fig. 2B,C, where CBA refers to clustered by 399 binding atoms, defined in Fig. 2A. We find that centroids 400 from different clustering methods vary in different structural 401 domains, Fig. 2C. The structural fluctuation around the binding ${ }_{402}$ site (facing the reader in Fig. 2C) are most likely to affect ${ }_{403}$ ligand binding. By restricting the set features input to the ${ }_{404}$ clustering workflow to the binding atoms, we find that the ${ }_{405}$ CBA methods capture increased variability in the binding 406 site.

\section{${ }_{407} 4.1$ Initial Docking}

408 Our FRED docking results performed worse than random rank 409 ordering (Fig. 3A). To investigate the influence of docking 410 algorithm, both scoring and conformational searching, we ${ }_{411}$ also performed docking with Schrodinger's Glide (81). The ${ }_{412}$ rank order correlation of the predictions from Glide docking ${ }_{413}$ were better than random rank ordering (Fig. 3B).

${ }_{414} \quad$ There are some inherent differences between OpenEye's ${ }_{415}$ FRED and Schrodinger's Glide conformational search algo${ }_{416}$ rithms. FRED's docking algorithm emphasizes shape com${ }_{417}$ plementarity between the ligand and protein through an ex${ }_{418}$ haustive pose search that samples multiple ligand positions. It 419 accounts for ligand rotations and scores multiple poses before 420 selecting one top scoring pose per ligand (46). On the other ${ }_{421}$ hand, Glide's docking algorithm begins with receptor grid ${ }_{422}$ generation and focuses on ligand binding energy, including ${ }_{423}$ a ligand minimization with a standard molecular mechanics ${ }_{424}$ energy function, the OPLS-AA force field, and a distance${ }_{425}$ dependent dielectric model. In addition, the final poses are ${ }_{426}$ refined with a Monte-Carlo procedure to find torsional min${ }_{427}$ ima (47). Both methods consider ligand conformers, either ${ }_{428}$ generated separately (through OpenEye OMEGA) or as part 429 of the docking workflow (Glide).

The scoring functions also differ between the two software. ${ }_{431}$ For FRED, the ChemGauss 4 scoring function, which uses ${ }_{432}$ Gaussian-smoothed step-function based interaction potentials, ${ }_{433}$ is used to optimize top poses from the filtering steps (46). ${ }_{434}$ Meanwhile, Glide's GlideScore uses more complex and varied 435 weight functions for the various potential terms (47). The ${ }_{436}$ more complex approach to fit empirical scoring functions used 437 by Glide may have improved the pose prediction similarity to ${ }_{438}$ cocrystal poses and the rank ordering accuracy. 
A.

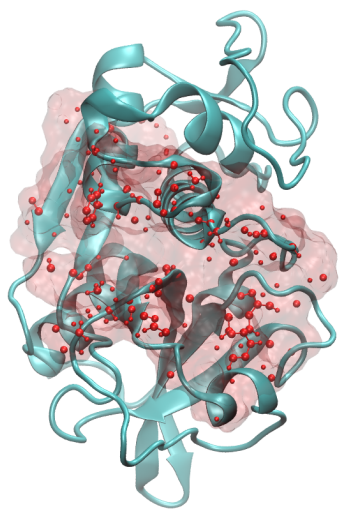

B.

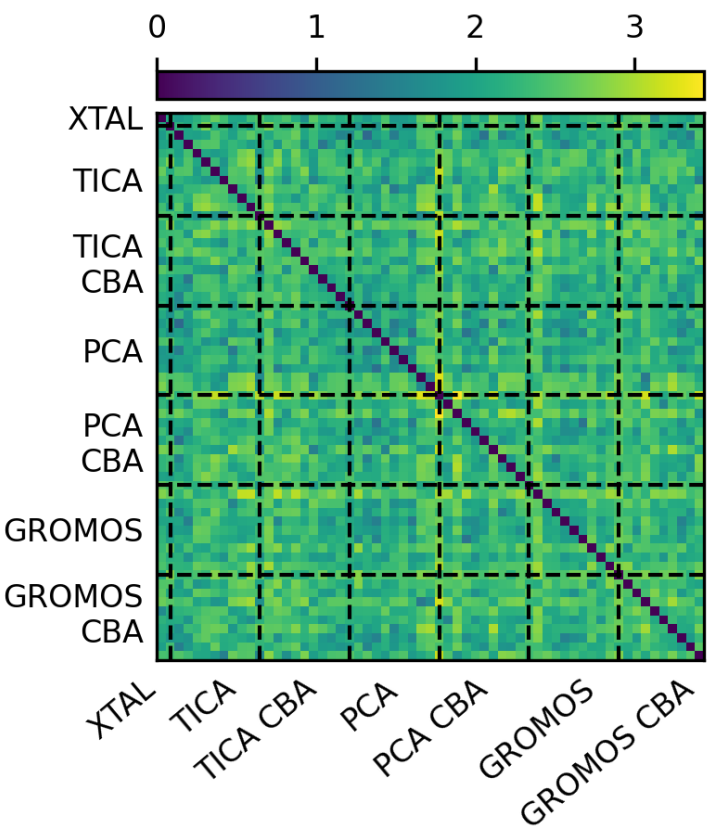

c.
TICA

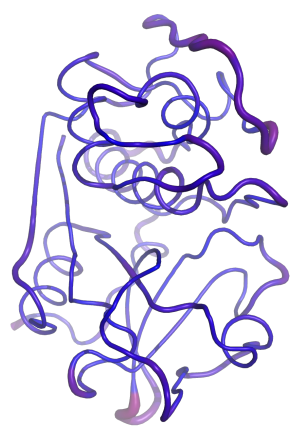

TICA CBA

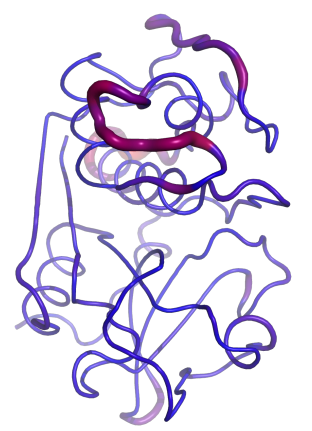

PCA

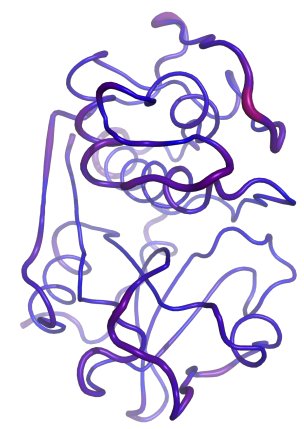

PCA CBA

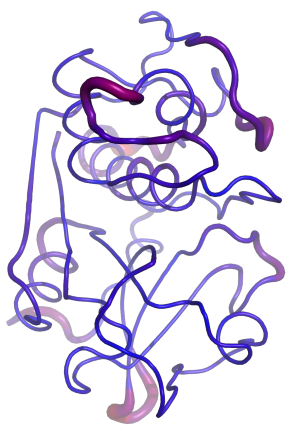

GROMOS

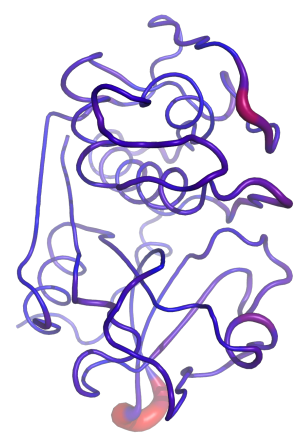

GROMOS CBA

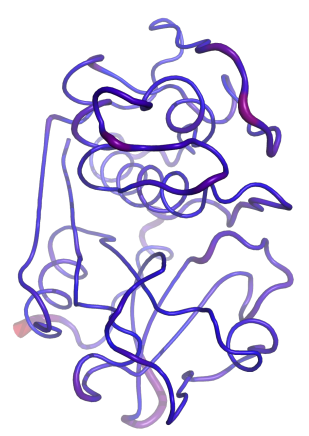

0

$\operatorname{RMSF}(\AA)$

Figure 2: Apo Molecular Dynamics (MD) Clustering Results. A) Binding Atoms definition for Clustered by Binding Atoms (CBA) centroids, defined by taking all atoms within $2 \AA$ of docked poses of a ligand from the D3R dataset (CatS_2) from both Glide and FRED SP apo blind docking. The crystal structure protein is depicted in NewCartoon and colored teal, while the binding atoms are both represented by red spheres and a transparent red surface representation, visualized in Visual Molecular Dynamics (VMD) (79, 80). B) The pairwise Root-Means-Squared-Deviations (RMSDs) of the binding atoms of the crystal structure and all 10 centroid structures from each clustering method are depicted in a heatmap. The centroids obtained from clustering have a range of RMSDs and therefore have structural variability. C) MD clustering extracts various centroid structures, and different clustering methods yield different conformations. The RMSF of the 10 centroids extracted from each clustering method, shown as the relative thickness and color, was calculated with MDTraj (68) and visualized using PyMOL (70). The orientation of the protein for parts $\mathrm{A}$ and $\mathrm{C}$ are the same. 
Kendall's $\tau$ For All Ligand Rankings

\begin{tabular}{|c|c|c|c|c|c|c|c|c|}
\hline \multirow[t]{2}{*}{ Docking Function } & \multirow[t]{2}{*}{ Scoring Method } & \multicolumn{7}{|c|}{ Clustering Methods } \\
\hline & & XTAL & TICA & PCA & GROMOS & TICA CBA & PCA CBA & GROMOS CBA \\
\hline \multirow{3}{*}{ FRED AB } & Minimum & -0.08 & 0.04 & -0.18 & -0.06 & -0.06 & -0.05 & -0.08 \\
\hline & W. Avg. & - & -0.11 & -0.20 & -0.04 & -0.11 & -0.09 & -0.06 \\
\hline & Avg. & - & -0.13 & -0.09 & -0.10 & -0.09 & -0.21 & -0.10 \\
\hline \multirow{3}{*}{$\begin{array}{l}\text { Glide } \\
\text { SP-AB }\end{array}$} & Minimum & 0.20 & 0.18 & 0.18 & 0.22 & 0.12 & 0.28 & 0.12 \\
\hline & W. Avg. & - & 0.21 & 0.20 & 0.18 & 0.17 & 0.24 & 0.21 \\
\hline & Avg. & - & 0.20 & 0.21 & 0.25 & 0.20 & 0.24 & 0.23 \\
\hline \multirow{3}{*}{$\begin{array}{l}\text { Glide } \\
\text { SP-AR }\end{array}$} & Minimum & 0.13 & 0.14 & 0.13 & 0.13 & 0.11 & 0.11 & 0.09 \\
\hline & W. Avg. & - & 0.08 & 0.07 & 0.10 & 0.09 & 0.05 & 0.07 \\
\hline & Avg. & - & 0.12 & 0.07 & 0.09 & 0.07 & 0.06 & 0.09 \\
\hline \multirow{3}{*}{$\begin{array}{l}\text { Glide } \\
\text { XP-AB }\end{array}$} & Minimum & 0.20 & 0.11 & 0.11 & 0.12 & 0.11 & 0.24 & 0.14 \\
\hline & W. Avg. & - & 0.10 & 0.08 & 0.08 & 0.11 & 0.17 & 0.15 \\
\hline & Avg. & - & 0.11 & 0.08 & 0.07 & 0.12 & 0.19 & 0.17 \\
\hline \multirow{3}{*}{$\begin{array}{l}\text { Glide } \\
\text { XP-AR }\end{array}$} & Minimum & 0.13 & 0.14 & 0.10 & 0.12 & 0.11 & 0.09 & 0.13 \\
\hline & W. Avg. & - & 0.10 & 0.07 & 0.04 & 0.07 & 0.03 & 0.11 \\
\hline & Avg. & - & 0.11 & 0.07 & 0.06 & 0.04 & 0.04 & 0.08 \\
\hline \multirow{3}{*}{$\begin{array}{l}\text { Glide } \\
\text { SP-HB }\end{array}$} & Minimum & 0.09 & 0.17 & 0.14 & 0.18 & 0.23 & 0.23 & 0.18 \\
\hline & W. Avg. & - & 0.20 & -0.01 & 0.17 & 0.24 & 0.14 & 0.20 \\
\hline & Avg. & - & 0.22 & -0.01 & 0.21 & 0.23 & 0.18 & 0.21 \\
\hline \multirow{3}{*}{$\begin{array}{l}\text { Glide } \\
\text { SP-HR }\end{array}$} & Minimum & 0.12 & 0.18 & 0.13 & 0.11 & 0.16 & 0.17 & 0.15 \\
\hline & W. Avg. & - & 0.13 & 0.09 & 0.08 & 0.15 & 0.11 & 0.13 \\
\hline & Avg. & - & 0.15 & 0.11 & 0.12 & 0.14 & 0.13 & 0.14 \\
\hline
\end{tabular}

Table 1: The Kendall's $\tau$ s for the FRED and initial Glide docking show slight fluctuations in different scoring schemes, but do not show any immense improvement. Here we show the Kendall's $\tau$ from rank orderings produced through various docking functions, clustering methods, and scoring schemes. Docking Functions are labeled accordingly: SP: Glide Standard Precision Docking, XP: Glide Extra Precision Docking; A: apo structure, H: holo structure; B: blind docking, R: restrained docking. We experimented with these scoring schemes to test if a particular method of discerning scores for each ensemble would better represent the protein binding mechanisms and improve rank ordering. The various scoring schemes were the Minimum, Weighted Average (W. Avg.), and Average (Avg.). 
A.

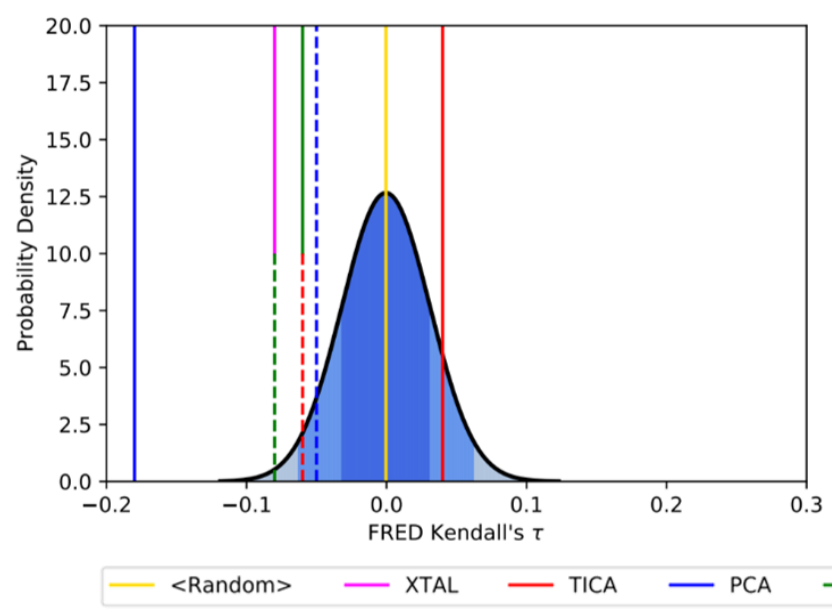

B.

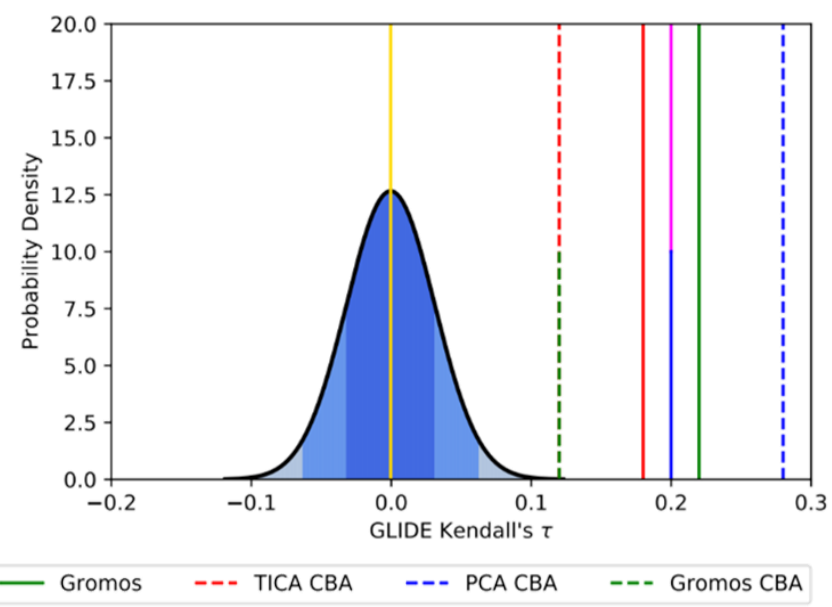

Figure 3: The FRED rank ordering results were unsuccessful in producing a higher Kendall's $\tau$ value than random ordering, while the Glide results were able to predict better than random. A) Kendall's $\tau$ values for ligand rankings based on minimum scores from OpenEye's FRED blind docking to apo MD centroids, compared to a random rank ordering distribution. B) Kendall's $\tau$ values for ligand rankings based on minimum scores from Schrodinger's Glide docking to apo MD centroids, compared to a random rank ordering distribution. In both A) and B) a probability distribution function is graphed from the Kendall's $\tau$ values of 10,000 random ligand rank orderings. The distribution has $\mu=0$ and $\sigma=0.031$.

\section{${ }_{439} 4.2$ Scoring Scheme Results}

${ }_{440}$ Next, we investigated if the approach to compute a single ${ }_{441}$ score from an ensemble of scores can improve the accuracy ${ }_{442}$ of our predictions. There are several ways to obtain a single ${ }_{443}$ score from an ensemble of values. The first is to take the ${ }_{444}$ minimum score of the ensemble. This assumes that the other ${ }_{445}$ configurations do not contribute to the ligand binding energy. ${ }_{446}$ Relaxing this assumption, it is possible to consider the contri${ }_{447}$ butions of other receptor configurations by using an average 448 or weighted average of the ensemble values. The choice of 449 weights may be assigned by the probability of observing each 450 conformation among other strategies. Limitations from the 451 limited sampling of MD may lead to unintended biases in the 452 ensemble weights.

${ }_{453}$ In our results, we saw minor fluctuations in Kendall's ${ }_{454} \tau \mathrm{s}$ across different scoring schemes (Table 1). While some ${ }_{455}$ conditions saw improvements to Kendall's $\tau$ when using the 456 weighted average versus the minimum score, no consistent ${ }_{457}$ rationale for these improvements were found. It is therefore ${ }_{458}$ unclear from this system and study whether or not incorpo${ }_{459}$ rating receptor flexibility can improve predictions of rank 460 ordered correlation. We hypothesize that the challenges of ${ }_{461}$ docking to CatS which has a large solvent-exposed binding ${ }_{462}$ pocket may outweigh the benefits of incorporating receptor ${ }_{463}$ flexibility which has been reported in other works $(26,30)$.

${ }_{464}$ To further understand the shortcomings in our approach, ${ }_{465}$ we conducted multiple revisions to both the trajectory cluster466 ing and the docking methodology.

\section{${ }_{467}$ 4.3 Pose Analysis and Glide Docking Revisions}

${ }_{469}$ We found that the ligands in the CatS dataset had a common 470 tetrahydropyrido-pyrazole core to other ligands with published ${ }_{471}$ cocrystal structures from a prior D3R Grand Challenge (GC3) ${ }_{472}$ (Fig. S1) $(7,50)$. The poses from FRED docking were varied ${ }_{473}$ and often located opposite from the binding location of similar ${ }_{474}$ cocrystallized ligands (Fig. 4A). Other cocrystals contain 475 ligands bound to this alternative site, although these ligands 476 are dissimilar to the ones in our dataset (ligands 29 to 48 in 477 Fig. S1, Table. S1) (82).

${ }_{478}$ Glide docking produced some poses similar to the cocrys479 tal pose (Fig. 4B) although it also produced more unexpected ${ }_{480}$ poses. We also observed cocrystals with ligands binding in the ${ }_{481}$ less common "flipped core" configuration, shown in Fig. 4C, 482 reported in $\mathrm{GC} 3(7)$.

To test the hypothesis whether improved pose similarity 484 to cocrystal structures can improve docking accuracy, we 485 applied a distance-restraint to the common core of the ligands 486 using the core position of published cocrystals with similar ${ }_{487}$ ligands as a reference point (Fig. 4D). Other work has found 488 that approaches which use information from cocrystals such 489 as template docking or restraints can improve pose accuracy $490(5-7,83,84)$. The restraint employed eliminated poses which ${ }_{491}$ deviate significantly from the cocrystal pose while permitting 492 the flipped configuration. As shown in Fig. 4E, the RMSDs ${ }_{493}$ of the tetrahydropyrido-pyrazole core in SP apo docking were 494 reduced (from a median of $7.39 \AA$ to $1.40 \AA$ A ) by adding the ${ }_{495}$ restraint, however, this did not improve the accuracy of the 

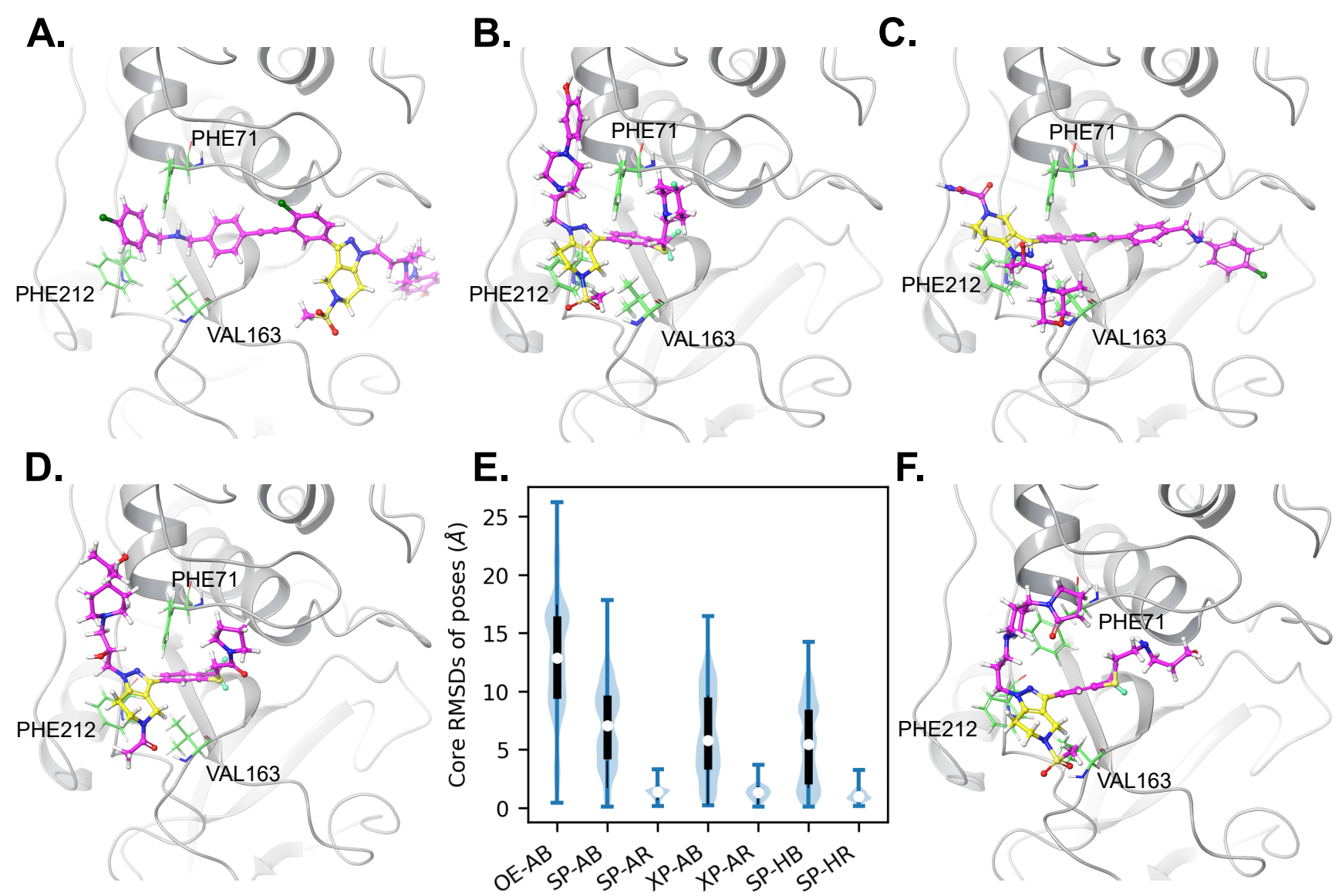

Figure 4: Docking pose analysis shows that a distance-restraint improves pose accuracy. A) Ligand CatS 259, example of an inaccurate FRED pose, with the core in a different location than the crystal structure of a similar ligand. B) Ligand CatS 118 of the SP apo blind crystal docking: ideal pose most similar to the cocrystal structure. C) Ligand CatS 363 of the SP apo blind crystal docking: some docked ligands show a flipped core binding mode that is less common but can be found in some available cocrystals. (7). D) cocrystal pose (PDBID: 5QC4 (11)) Ligand carbons are pink; ligand common core carbons are yellow; key binding residues PHE71, VAL163, and PHE212 are green. E) The RMSDs of the ligand core for each pose in each Glide docking method show that blind poses were concentrated farther from the cocrystal position compared to the ligand-core-restrained docking. In addition, the FRED average core RMSD is larger than that of all blinded Glide RMSDs. Each violin is composed of all minimum poses for each clustering method which contributed to the final rank ordering and the crystal structure poses, totaling $n=3213$ per violin. Method Acronyms: OE: OpenEye FRED docking, SP: Glide Standard Precision Docking, XP: Glide Extra Precision Docking; A: apo structure, H: holo structure; B: blind docking, R: restrained docking. The median is represented in white, the interquartile range is shown in black, and the minimum and maximum values are shown as whiskers. F) Ligand CatS 23 of the SP apo restrained PCA docking: When the ligand is restrained, it can be unnaturally docked in receptors that are dissimilar to the cocrystal, such as here where the PHE71 is in a different configuration. 
496 ranking (Table 1$)$.

497 The ligand core restraint may not be appropriate for all 498 centroids (e.g., Fig. 4F). The reference for the restraint is 499 defined for all receptor configurations by RMSD alignment to 500 the cocrystal structure. Receptor configurations which exhibit 501 large structural differences from the cocrystal structure may 502 have poor binding site alignment which introduces uncertainty ${ }_{503}$ into the approach. For some receptor configurations, restraints ${ }_{504}$ lead to atypical binding poses with high solvent accessibility.

505 To test whether using a more complex scoring function and 506 search algorithm at the cost of computational efficiency can 507 improve the predicted rank ordering, we compared between ${ }_{508}$ GlideScore SP and GlideScore Extra Precision (XP), with 509 and without the restraint, using the same ensembles from apo ${ }_{510}$ MD. Compared to Glide SP, Glide XP (i) has more exhaustive 511 docking by performing Glide SP docking then performing a 512 separate anchor-and-grow sampling procedure, and (ii) the ${ }_{513}$ Glide XP scoring function penalizes ligand poses more harshly 514 with desolvation penalties, identification of enhanced binding 515 motifs, and higher receptor-ligand shape complementarity 516 (76). Glide XP has been found to outperform other methods 517 and achieve better drug discovery results than Glide SP (81). ${ }_{518}$ We found that Glide XP did not improve our predictions (1). ${ }_{519}$ Although the poses predicted by XP were more similar to the ${ }_{520}$ cocrystallized poses (4E).

${ }_{521}$ To test whether conformational selection may lead to ${ }_{522}$ improved results, we docked to centroids picked from a holo ${ }_{523}$ MD simulation. McGovern and Shoichet have showed that use ${ }_{524}$ of a holo structure can improve enrichment of lead compound ${ }_{525}$ identification (85). We also expected that structures with a 526 ligand would lead to lower ligand core RMSD's with more ${ }_{527}$ accurate active residue positioning. However, the Kendall's ${ }_{528} \tau \mathrm{s}$ of the rank ordering stayed within the same range as the ${ }_{529}$ original apo docking, even when the ligand was restrained 530 (1). Upon further analysis of the structural fluctuations of the 531 apo and holo MD centroids, we find that residue PHE71 is ${ }_{532}$ restricted by the ligand while other regions of the binding ${ }_{533}$ pocket exhibited similar structural variability (Fig. S2). When ${ }_{534}$ ligands were blindly docked using Glide SP to the holo ${ }_{535}$ structures, the resulting poses remained different than the ${ }_{536}$ cocrystal pose. The average RMSD of the docked ligand cores ${ }_{537}$ was $5.47 \AA$ from the core of the cocrystal ligand (4C). Overall, ${ }_{538}$ the blind docking to structure from the holo MD trajectory had 539 a slightly lower ligand core RMSD compared to the results ${ }_{540}$ form docking to the apo MD (Fig. S3). When a core restraint ${ }_{541}$ was applied upon docking to configurations from the holo 542 trajectory, even with the influence of the bound ligand on the ${ }_{543}$ binding site, the rank ordering did not improve (1).

$544 \quad$ Although others have suggested that improved poses could ${ }_{545}$ yield better scores (86), we found that improvements to the ${ }_{546}$ predicted poses from the application of ligand restraints and/or ${ }_{547}$ docking to holo receptor conformations did not improve our ${ }_{548}$ predictions. This suggests that there may be other confounding 549 factors influencing our results.

\section{${ }_{550} 5$ CONCLUSION}

${ }_{551}$ In this work we describe our submission to subchallenge 2 ${ }_{552}$ of the Drug Design Data Resource (D3R) Grand Challenge ${ }_{553} 4$ where we performed ensemble docking to rank order lig${ }_{554}$ ands by binding affinity. We explore and compare several 555 factors including the choice of clustering algorithm for choos556 ing representative receptor conformations and two docking 557 workflows with and without restraints to improve pose accu558 racy. The different clustering algorithms produce different 559 structural ensembles which can influence the docking results. ${ }_{560}$ Owing to the difficulty of docking to the CatS system, which ${ }_{561}$ has been recognized by others (87), we find that more so562 phisticated approaches can improve rank ordering compared 563 to naive settings produced by FRED and GLIDE using a 564 basic ensemble docking workflow (7). Glide yielded better 565 rank order correlations than FRED although no notable dif566 ferences between the clustering algorithms was observed. We 567 conclude that confounding factors and complications of the ${ }_{568}$ CatS system outweigh the benefits of ensemble docking. We 569 explored if rank-order correlation could be improved with 570 better pose accuracy by performing docking with restraints 571 in addition to docking with receptor conformations extracted 572 from a holo trajectory with ligand removed. We find that both 573 approaches improve the pose similarity of docked ligands to 574 related cocrystallized ligands, but do not improve the rank 575 order correlation.

This project illustrates the benefits of partnering with high 577 school and undergraduate students to participate in commu578 nity challenges. Grand challenges are excellent resources for 579 teaching research skills through a semi-guided, goal-oriented ${ }_{580}$ project, with expert curated datasets and deadlines. The stu${ }_{581}$ dents were exposed to important research skills, such as 582 managing time, selecting and performing data analyses, and 583 making publication-quality figures, at early stages of their ${ }_{584}$ scientific career. Owing to the computational nature of this 585 challenge, the students also gained experience with data man${ }_{586}$ agement, computational thinking, and script development. We ${ }_{587}$ suggest that student participation in community challenges 588 can benefit both the community and the students and hope 589 this work encourages others to explore this approach.

\section{AUTHOR CONTRIBUTIONS}

${ }_{591}$ Conceptualization, B.C.T., B.R.J., C.T.L. and R.E.A.; Soft592 ware, J.L.G., D.K., and C.C.; Investigation, J.L.G., D.K., and ${ }_{593}$ C.C.; Resources, B.C.T., B.R.J., C.T.L. and R.E.A.; Writ594 ing - Original Draft, J.L.G, and D.K.; Writing - Review 595 and Editing, J.L.G, D.K., C.C., B.C.T., B.R.J., C.T.L. and ${ }_{596}$ R.E.A.; Visualization, J.L.G., D.K.; Supervision and Project ${ }_{597}$ Administration, B.C.T., B.R.J., C.T.L. and R.E.A.; Funding 598 Acquisition, R.E.A. 


\section{${ }_{599}$ ACKNOWLEDGMENTS}

600 This work is supported by the National Biomedical Computa- ${ }^{647}$ ${ }_{601}$ tion Resource NIH Grant P41-GM103426, and the National ${ }^{648}$ ${ }_{602}$ Science Foundation through The Extreme Science and Engi- ${ }^{649}$ ${ }_{603}$ neering Discovery Environment (XSEDE) supercomputing ${ }^{650}$ 604 resources provided via Award TG-CHE060073 to R.E.A. 651 ${ }_{605}$ C.T.L. is funded by a Hartwell Foundation Postdoctoral Fel- ${ }_{652}$ 606 lowship. We thank D3R and the organizers of Grand Challenge ${ }_{653}$ ${ }_{607} 4$ for hosting the challenge and reporting results. We would ${ }_{654}$ 608 also like to acknowledge Mason V. Holst, Gaurie Gunasekaran, 655 ${ }_{609}$ Gray Thoron, and Jeffery R. Wagner for their contributions to 656 610 preliminary work and/or helpful discussions.

\section{${ }_{611}$ SUPPLEMENTARY MATERIAL}

612 The structural similarity of the dataset ligands to cocrystal- 660 ${ }_{613}$ lized ligands, RMSF across receptor structures for the apo ${ }_{661}$ 614 and holo trajectories, and comparison of ligand core RMSD 662 615 across clustering methods are available in the supplemental ${ }_{663}^{63}$ 616 information.

\section{${ }_{617}$ REFERENCES}

1. Barbara M. Bolten and Tracy DeGregorio. "Trends in development cycles". In: Nature Reviews Drug Discovery 1.5 (2002), pp. 335-336. IssN: 1474-1784. DOI: 10.1038/ nrd805. URL: https://Www . nature. com/articles/ nrd805.

2. Steven M. Paul et al. "How to improve R\&D productivity: the pharmaceutical industry's grand challenge". In: Nature Reviews Drug Discovery 9.3 (2010), pp. 203214. ISSN: 1474-1784. DOI: $10.1038 / \operatorname{nrd3078.~URL:~}$ https: //www . nature. com/articles/nrd3078.

3. A. Lavecchia and C. Giovanni. "Virtual Screening Strate- 678 gies in Drug Discovery: A Critical Review". In: CMC 20.23 (2013), pp. 2839-2860. ISSN: 09298673. DOI: 10. 2174 / 09298673113209990001. URL: http : / / WWw . eurekaselect . com/openurl/content . php? genre $=$ article $\&$ issn $=0929-8673 \&$ volume $=20 \&$ issue $=23 \&$ spage $=2839$.

4. Evanthia Lionta et al. "Structure-based virtual screening for drug discovery: principles, applications and recent advances". In: Current Topics in Medicinal Chemistry ${ }_{687}$ 14.16 (2014), pp. 1923-1938. ISSN: 1873-4294. DOI: 10. 2174/1568026614666140929124445.

5. Symon Gathiaka et al. "D3R grand challenge 2015: Eval- 690 uation of proteinligand pose and affinity predictions". ${ }^{691}$ In: Journal of Computer-Aided Molecular Design 30.9 (2016), pp. 651-668. ISSN: 0920-654X, 1573-4951. DOI: ${ }_{693}$ 10.1007/s10822-016-9946-8. URL: http: / / link . 694 springer . com/10.1007/s10822-016-9946-8.
6. Zied Gaieb et al. "D3R Grand Challenge 2: blind prediction of protein-ligand poses, affinity rankings, and relative binding free energies". In: Journal of Computer-Aided Molecular Design 32.1 (2018), pp. 1-20. IssN: 1573-4951. DoI: $10.1007 / \mathrm{s} 10822-017-0088-4$.

7. Zied Gaieb et al. "D3R Grand Challenge 3: blind prediction of proteinligand poses and affinity rankings". In: Journal of Computer-Aided Molecular Design 33.1 (2019), pp. 1-18. ISSN: 0920-654X, 1573-4951. DOI: 10. 1007/s10822-018-0180-4. uRL: http: / / link. springer. com/10.1007/s10822-018-0180-4.

8. D3R $\mid$ Grand Challenge 4. uRL: https: //drugdesigndata. org/about/grand-challenge-4.

9. Robin L. Thurmond et al. "Identification of a Potent and Selective Noncovalent Cathepsin S Inhibitor". In: Journal of Pharmacology and Experimental Therapeutics 308.1 (2004), pp. 268-276. ISSN: 0022-3565, 1521-0103. DoI: 10. 1124 / jpet. 103. 056879. uRL: http : / / jpet . aspetjournals . org/lookup/doi/10.1124/jpet . 103.056879.

10. Michael K. Ameriks et al. "Diazinones as P2 replacements for pyrazole-based cathepsin S inhibitors". In: Bioorganic \& Medicinal Chemistry Letters 20.14 (2010), pp. 40604064. ISSN: 0960894X. DOI: 10.1016/j . bmcl . 2010. 05. 086. uRL: https: //linkinghub.elsevier.com/ retrieve/pii/s0960894X10007274.

11. Danielle K. Wiener et al. "Thioether acetamides as P3 binding elements for tetrahydropyrido-pyrazole cathepsin S inhibitors". In: Bioorganic \& Medicinal Chemistry Letters 20.7 (2010), pp. 2379-2382. Issn: 0960894X. DOI: $10.1016 / \mathrm{j}$. bmcl . 2010.01.103. URL: https : / / linkinghub . elsevier . com / retrieve / pii / S0960894X10001150.

12. Michael K. Ameriks et al. "Pyrazole-based cathepsin S inhibitors with arylalkynes as P1 binding elements". In: Bioorganic \& Medicinal Chemistry Letters 19.21 (2009), pp. 6131-6134. IssN: 0960894X. DOI: 10.1016/ j . bmcl . 2009.09.014. uRL: https: / / linkinghub . elsevier.com/retrieve/pii/S0960894X09012712.

13. Rommie E. Amaro et al. "Discovery of drug-like inhibitors of an essential RNA-editing ligase in Trypanosoma brucei". In: Proceedings of the National Academy of Sciences of the United States of America 105.45 (2008), pp. 17278-17283. ISSN: 0027-8424. DOI: 10.1073/ pnas. 0805820105. uRL: https: / / wWW . ncbi . nlm . nih.gov/pmc/articles/PMC2577703/.

14. Jin Li, Ailing Fu, and Le Zhang. "An Overview of Scoring Functions Used for ProteinâLigand Interactions in Molecular Docking". In: Interdisciplinary Sciences: Computational Life Sciences 11.2 (2019), pp. 320-328. ISSN: 1913-2751, 1867-1462. DOI: 10.1007/s12539-01900327 - w. URL: http: / / link. springer . com/10 . 1007/s12539-019-00327-w. 
699 15. Sheng-You Huang, Sam Z. Grinter, and Xiaoqin Zou. 748 23. Stefano Gianni, Jakob Dogan, and Per Jemth. "Distin-

"Scoring functions and their evaluation methods for pro- 749 teinâligand docking: recent advances and future direc- 750 tions". In: Physical Chemistry Chemical Physics 12.40751 (2010), p. 12899. IssN: 1463-9076, 1463-9084. DOI: 752 10. 1039 / c0cp00151a. URL: http : / / xlink. rsC. 753 org/?DOI=cOcp00151a.

16. M. Teodoro and L. Kavraki. "Conformational Flexibility 755 Models for the Receptor in Structure Based Drug De- ${ }_{756}$ sign”. In: Current Pharmaceutical Design 9.20 (2003), 757 pp. 1635-1648. ISSN: 13816128. DOI: 10.2174/13816120353 URL: http: / / wWw. eurekaselect. com/openurl/ 759 content . php? genre $=$ article\&issn $=1381-6128 \& 760$ volume $=9 \&$ issue $=20 \&$ spage $=1635$.

17. Gregory Sliwoski et al. "Computational Methods in Drug 762 Discovery". In: Pharmacological Reviews 66.1 (2014), 763 pp. 334-395. ISSN: 0031-6997. DoI: 10.1124/pr . 112 - 764 Q07336. URL: https: / / wWw . ncbi .nlm.nih.gov/ pmc/articles/PMC3880464/.

18. Mark von Itzstein et al. "Rational design of potent ${ }_{767}$ sialidase-based inhibitors of influenza virus replication". 768 In: Nature 363.6428 (1993), pp. 418-423. ISSN: 0028- 769 0836, 1476-4687. DoI: 10.1038/363418a0. URL: http: 770 //www . nature. com/articles/363418a0.

19. Maxim Totrov and Ruben Abagyan. "Flexible ligand $7_{2}$ docking to multiple receptor conformations: a prac- 773 tical alternative". In: Current Opinion in Structural 774 Biology 18.2 (2008), pp. 178-184. ISSN: 0959440X. 775 DOI: $10.1016 / \mathrm{j}$. sbi.2008.01.004. URL: https : 776 / / linkinghub . elsevier . com/retrieve / pii / 777 S0959440X08000080.

\section{8}

20. Nataraj S. Pagadala, Khajamohiddin Syed, and Jack 77928 Tuszynski. "Software for molecular docking: a review". 780 In: Biophysical Reviews 9.2 (2017), pp. 91-102. ISSN: 781 1867-2450. DOI: 10.1007/s12551-016-0247-1. URL: 782 https://wWw.ncbi.nlm.nih.gov/pmc/articles/ 783 PMC5425816/.

21. R Elber and M Karplus. "Multiple conformational states 785 of proteins: a molecular dynamics analysis of myoglobin". 786 In: Science 235.4786 (1987), pp. 318-321. IsSN: 0036- 787 8075, 1095-9203. DoI: 10.1126/science.3798113. 788 URL: https://www. sciencemag.org/lookup/doi/ 789 10.1126/science. 3798113.

22. Buyong Ma et al. "Folding funnels and binding mecha- 791 nisms". In: Protein Engineering, Design and Selection 792 12.9 (1999), pp. 713-720. IssN: 1741-0134, 1741-0126. 793 DoI: $10.1093 /$ protein / 12.9 .713 . URL: https : 794 // academic oup . com/peds / article-lookup / 795 doi/10.1093/protein/12.9.713. guishing induced fit from conformational selection". In: Biophysical Chemistry 189 (2014), pp. 33-39. Issn: 03014622. DOI: $10.1016 / \mathrm{j}$. bpc. 2014.03 .003. URL: https: //linkinghub . elsevier . com/retrieve/ $\mathrm{pii} / \mathrm{S} 0301462214000428$.

24. Peter Csermely, Robin Palotai, and Ruth Nussinov. "Induced fit, conformational selection and independent dynamic segments: an extended view of binding events". In: Trends in biochemical sciences 35.10 (2010), pp. 5394545546. IssN: 0968-0004. DoI: 10.1016/j . tibs. 2010.04. Q09. URL: https://wwW . ncbi .nlm.nih.gov/pmc/ articles/PMC3018770/.

25. Rommie E. Amaro et al. "Ensemble Docking in Drug Discovery". In: Biophysical Journal 114.10 (2018), pp. 22712278. ISSN: 1542-0086. DOI: 10.1016/j . bpj . 2018 . 02 . Q38.

26. Tavina L. Offutt, Robert V. Swift, and Rommie E. Amaro. "Enhancing Virtual Screening Performance of Protein Kinases with Molecular Dynamics Simulations". In: Journal of Chemical Information and Modeling 56.10 (2016), pp. 1923-1935. ISSN: 1549-9596, 1549-960X. DoI: 10.1021/acs . jcim. 6b00261. URL: https: // pubs.acs.org/doi/10.1021/acs . jcim. 6b00261.

27. Heather A. Carlson, Kevin M. Masukawa, and J. Andrew McCammon. "Method for Including the Dynamic Fluctuations of a Protein in Computer-Aided Drug Design". In: The Journal of Physical Chemistry A 103.49 (1999), pp. 10213-10219. IsSN: 1089-5639, 1520-5215. DOI: 10.1021/jp991997z. URL: https://pubs .acs . org/doi/10.1021/jp991997z.

28. Heather A. Carlson et al. "Developing a Dynamic Pharmacophore Model for HIV-1 Integrase". In: Journal of Medicinal Chemistry 43.11 (2000), pp. 2100-2114. Isss: 0022-2623, 1520-4804. DOI: 10.1021/jm990322h. URL: https://pubs.acs.org/doi/10.1021/jm990322h.

${ }_{84}$ 29. Jung-Hsin Lin et al. "Computational Drug Design Accommodating Receptor Flexibility: The Relaxed Complex Scheme". In: Journal of the American Chemical Society 124.20 (2002), pp. 5632-5633. IssN: 0002-7863, 1520-5126. DOI: $10.1021 /$ ja0260162. URL: https : //pubs.acs.org/doi/10.1021/ja0260162.

190 30. Rommie E. Amaro, Riccardo Baron, and J. Andrew McCammon. "An improved relaxed complex scheme for receptor flexibility in computer-aided drug design". In: Journal of Computer-Aided Molecular Design 22.9 (2008), pp. 693-705. Issn: 0920-654X, 1573-4951. Dor: 10.1007/s10822-007-9159-2. uRL: http: / / link. springer. com/10.1007/s10822-007-9159-2. 
797 31. Sheng-You Huang and Xiaoqin Zou. "Ensemble docking 85040.

798 of multiple protein structures: Considering protein struc- 85 tural variations in molecular docking". In: Proteins: Struc- ${ }_{852}$ ture, Function, and Bioinformatics 66.2 (2006), pp. 399- ${ }_{853}$ 421. ISSN: 08873585. DOI: 10.1002 /prot. 21214 . URL: 854 http://doi.wiley.com/10.1002/prot.21214.

32. Lily S. Cheng et al. "Ensemble-based virtual screen- ${ }^{856}$ ing reveals potential novel antiviral compounds for avian ${ }^{857}$ influenza neuraminidase". In: Journal of Medicinal Chem- ${ }_{85}$ istry 51.13 (2008), pp. 3878-3894. ISSN: 1520-4804. DOI: 859 10.1021/jm8001197.

33. Jacob D. Durrant et al. "Novel Naphthalene-Based In- ${ }^{861}$ hibitors of Trypanosoma brucei RNA Editing Ligase 1". ${ }^{862}$ In: PLoS Neglected Tropical Diseases 4.8 (2010). ISSN: 863 1935-2727. Dor: 10.1371/ journal . pntd. 0000803. URL: https: // www . ncbi .nlm.nih.gov/pmc/ 865 articles/PMC2927429/.

34. Christopher D. Wassman et al. "Computational identifi- ${ }^{867}$ cation of a transiently open $\mathrm{L} 1 / \mathrm{S} 3$ pocket for reactivation 868 of mutant p53". In: Nature Communications 4 (2013), p. 1407. ISSN: 2041-1723. DoI: 10.1038/ncomms2361. URL: https : / / wWW . ncbi . nlm . nih. gov / pmc / articles/PMC3562459/.

35. Anthony Ivetac et al. "Discovery of Novel Inhibitors of HIV-1 Reverse Transcriptase Through Virtual Screening of Experimental and Theoretical Ensembles". In: Chemical biology \& drug design 83.5 (2014), pp. 521-531. ISSN: 1747-0277. DOI: $10.1111 /$ cbdd . 12277. URL: https://www.ncbi.nlm.nih.gov/pmc/articles/ PMC3999242/.

36. J. M. Barnard and G. M. Downs. "Clustering of chemical structures on the basis of two-dimensional similarity measures". In: J. Chem. Inf. Model. 32.6 (1992), pp. 644649. ISSN: 1549-9596. DOI: $10.1021 /$ ci00010a010. URL: https://pubs.acs.org/doi/abs/10.1021/ 88 ci00010a010.

37. Jianyin Shao et al. "Clustering Molecular Dynamics Trajectories: 1. Characterizing the Performance of Different Clustering Algorithms". In: Journal of Chemical Theory and Computation 3.6 (2007), pp. 2312-2334. Issn: 888 1549-9618, 1549-9626. DOI: 10.1021/ct700119m. URL: 889 https://pubs.acs.org/doi/10.1021/ct700119m. 890

38. Xiaohu Hu et al. "The dynamics of single protein molecules ${ }^{891}$ is non-equilibrium and self-similar over thirteen decades ${ }^{892}$ in time". In: Nature Physics 12.2 (2016), pp. 171-174. 89348 ISSN: 1745-2481. DOI: 10.1038/nphys3553. URL: https : 894 //www . nature. com/articles/nphys3553.

895

39. Edward Lyman and Daniel M. Zuckerman. "Ensemble- ${ }^{896}$ Based Convergence Analysis of Biomolecular Trajec- ${ }^{897}$ tories". In: Biophysical Journal 91.1 (2006), pp. 164- ${ }^{898}$ 172. ISSN: 0006-3495. Dor: 10.1529/biophysj . 106. 082941. URL: https: / / wwW . ncbi . nlm.nih.gov/ pmc/articles/PMC1479051/.
40. Wilfredo Evangelista Falcon et al. "Ensemble Docking in Drug Discovery: How Many Protein Configurations from Molecular Dynamics Simulations are Needed To Reproduce Known Ligand Binding?" In: The Journal of Physical Chemistry B 123.25 (2019), pp. 5189-5195. ISSN: 1520-6106, 1520-5207. Dor: 10.1021/acs . jpcb. 8b11491. URL: https: // pubs . acs. org/doi / 10 . 1021/acs. jpcb. 8b11491.

41. Martin K. Scherer et al. "PyEMMA 2: A Software Package for Estimation, Validation, and Analysis of Markov Models". In: J. Chem. Theory Comput. 11.11 (2015), pp. 5525-5542. ISSN: 1549-9618, 1549-9626. DOI: 10 . 1021/acs. jctc. 5b00743. uRL: https: //pubs . acs. org/doi/10.1021/acs. jctc. 5b00743.

42. J. A. Hartigan and M. A. Wong. "Algorithm AS 136: A KMeans Clustering Algorithm". In: Applied Statistics 28.1 (1979), p. 100. ISSN: 00359254. DoI: 10.2307/2346830. URL: https: / /www. jstor . org/stable/10.2307/ 2346830?origin=crossref.

43. Xavier Daura et al. "Peptide Folding: When Simulation Meets Experiment". In: (), p. 5.

${ }_{71}$ 44. Guillermo Perez-Hernandez et al. "Identification of slow molecular order parameters for Markov model construction". In: The Journal of Chemical Physics 139.1 (2013), p. 015102. ISSN: 0021-9606, 1089-7690. DOI: 10.1063/ 1.4811489. urL: http://aip. scitation.org/doi/ $10.1063 / 1.4811489$.

45. Charles C. David and Donald J. Jacobs. "Principal component analysis: a method for determining the essential dynamics of proteins". In: Methods in Molecular Biology (Clifton, N.J.) 1084 (2014), pp. 193-226. ISSN: 1940-6029. DOI: 10.1007/978-1-62703-658-0_11.

46. Mark McGann. "FRED Pose Prediction and Virtual Screening Accuracy". In: Journal of Chemical Information and Modeling 51.3 (2011), pp. 578-596. Issn: 1549-9596, 1549-960X. DOI: 10.1021/ci 100436p. URL: https://pubs.acs.org/doi/10.1021/ci100436p.

47. Richard A. Friesner et al. "Glide: A New Approach for Rapid, Accurate Docking and Scoring. 1. Method and Assessment of Docking Accuracy". In: Journal of Medicinal Chemistry 47.7 (2004), pp. 1739-1749. Issn: 0022-2623, 1520-4804. DOI: 10. 1021/jm0306430. URL: https://pubs.acs.org/doi/10.1021/jm0306430.

48. Cynthia Gray et al. "Known Structure, Unknown Function: An Inquiry-Based Undergraduate Biochemistry Laboratory Course: Known Structure, Unknown Function: A New Biochemistry Lab". en. In: Biochemistry and Molecular Biology Education 43.4 (2015), pp. 245-262. IssN: 14708175. DOI: $10.1002 / \mathrm{bmb} .20873$. 
${ }_{899}$ 49. Jennifer M. Heemstra et al. "Throwing Away the Cook- 947 58. Mats H. M. Olsson et al. "PROPKA3: Consistent Treat-

900 book: Implementing Course-Based Undergraduate Re- 948

901 search Experiences (CUREs) in Chemistry". In: Educa- 949

$902 \quad$ tional and Outreach Projects from the Cottrell Scholars 950 ${ }_{903} \quad$ Collaborative Undergraduate and Graduate Education ${ }_{951}$

$904 \quad$ Volume 1. Vol. 1248. ACS Symposium Series. American 905 Chemical Society, 2017. Chap. 3, pp. 33-63. IsBN: 978-

${ }_{912}$ 51. Maestro. New York, NY, 2019.

${ }_{913}$ 52. G. Madhavi Sastry et al. "Protein and ligand preparation: 914 0-8412-3208-2. Dor: 10.1021/bk-2017-1248. ch003.

50. Helen M. Berman et al. "The Protein Data Bank". In: 955 Nucleic Acids Research 28.1 (2000), pp. 235-242. ISSN: 956 0305-1048. Dor: 10.1093/nar/28.1.235. URL: https: 957 //academic . oup . com/nar/article/28/1/235/958 2384399.

\section{9}

960

961 parameters, protocols, and influence on virtual screening enrichments". In: Journal of Computer-Aided Molecular Design 27.3 (2013), pp. 221-234. ISSN: 0920-654X, 15734951. DoI: $10.1007 /$ s10822-013-9644-8. URL: http: 96 / / link. springer. com / 10.1007 / s10822-013-966 9644-8.

53. Junmei Wang et al. "Development and testing of a general amber force field”. In: J. Comput. Chem. 25.9 (2004), pp. 1157-1174. ISSN: 0192-8651, 1096-987X. DOI: 10 . 1002 / jcc. 20035. urL: http: //doi .wiley. com/10. $1002 / j c c .20035$.

54. Christopher I. Bayly et al. "A Well-Behaved Electrostatic Potential Based Method Using Charge Restraints for Deriving Atomic Charges: The RESP Model". In: The Journal of Physical Chemistry 97.40 (1993), pp. 1026910280. ISSN: 0022-3654. DOI: 10.1021/j100142a004.

55. Araz Jakalian et al. "Fast, Efficient Generation of HighQuality Atomic Charges. AM1-BCC Model: I. Method". en. In: Journal of Computational Chemistry 21.2 (2000), pp. 132-146. ISSN: 1096-987X. DOI: 10.1002/(SICI) $1096-987 \mathrm{X}(20000130) 21: 2<132::$ AID - JCC5 $>3.0$. $\mathrm{CO} ; 2-\mathrm{P}$.

56. Araz Jakalian, David B. Jack, and Christopher I. Bayly. "Fast, Efficient Generation of High-Quality Atomic Charges. AM1-BCC Model: II. Parameterization and Validation". eng. In: Journal of Computational Chemistry 23.16 (2002), pp. 1623-1641. ISSN: 0192-8651. DOI: 10.1002/ 988 jcc. 10128 .

57. Chresten R. Søndergaard et al. "Improved Treatment of Ligands and Coupling Effects in Empirical Calculation and Rationalization of pKa Values". In: Journal of Chemical Theory and Computation 7.7 (2011), pp. 2284-2295. ISSN: 1549-9618. DOI: 10.1021/ct200133y. ment of Internal and Surface Residues in Empirical pKa Predictions". In: Journal of Chemical Theory and Computation 7.2 (2011), pp. 525-537. ISSN: 1549-9618. DOI: $10.1021 /$ ct100578z.

59. D.A. Case, I.Y. Ben-Shalom, S.R. Brozell, D.S. Cerutti, T.E. Cheatham, III, V.W.D. Cruzeiro, T.A. Darden, R.E. Duke, D. Ghoreishi, M.K. Gilson, H. Gohlke, A.W. Goetz, D. Greene, R Harris, N. Homeyer, Y. Huang, S. Izadi, A. Kovalenko, T. Kurtzman, T.S. Lee, S. LeGrand, P. Li, C. Lin, J. Liu, T. Luchko, R. Luo, D.J. Mermelstein, K.M. Merz, Y. Miao, G. Monard, C. Nguyen, H. Nguyen, I. Omelyan, A. Onufriev, F. Pan, R. Qi, D.R. Roe, A. Roitberg, C. Sagui, S. Schott-Verdugo, J. Shen, C.L. Simmerling, J. Smith, R. SalomonFerrer, J. Swails, R.C. Walker, J. Wang, H. Wei, R.M. Wolf, X. Wu, L. Xiao, D.M. York and P.A. Kollman. AMBER 2018. University of California, San Francisco, 2018.

60. Jeremy D. Schmit et al. "SLTCAP: A Simple Method for Calculating the Number of Ions Needed for MD Simulation". In: Journal of Chemical Theory and Computation 14.4 (2018), pp. 1823-1827. IssN: 1549-9618, 1549-9626. DoI: $10.1021 /$ acs . jctc. 7b01254. uRL: https: / / pubs.acs.org/doi/10.1021/acs. jctc.7b01254.

61. Chad W. Hopkins et al. "Long-Time-Step Molecular Dynamics through Hydrogen Mass Repartitioning”. In: Journal of Chemical Theory and Computation 11.4 (2015). Publisher: American Chemical Society, pp. 18641874. ISSN: 1549-9618. DOI: 10.1021/ct5010406. URL: https://doi.org/10.1021/ct5010406.

62. Jean-Paul Ryckaert, Giovanni Ciccotti, and Herman J. C Berendsen. "Numerical Integration of the Cartesian Equations of Motion of a System with Constraints: Molecular Dynamics of n-Alkanes". en. In: Journal of Computational Physics 23.3 (1977), pp. 327-341. ISsN: 0021-9991. DoI: 10. 1016/0021-9991(77)90098-5.

63. Aapo Hyvarinen, Juha Karhunen, and Erkki Oja. Independent component analysis. New York: J. Wiley, 2001. 481 pp. ISBN: 978-0-471-40540-5.

64. L. Molgedey and H. G. Schuster. "Separation of a mixture of independent signals using time delayed correlations". In: Phys. Rev. Lett. 72.23 (1994), pp. 3634-3637. ISSN: 0031-9007. DoI: 10.1103/PhysRevLett . 72.3634. URL: https : / / link . aps . org / doi / 10.1103 / PhysRevLett. 72.3634.

65. Hao Wu and Frank NoÃl'. "Variational Approach for Learning Markov Processes from Time Series Data". In: J Nonlinear Sci 30.1 (2020), pp. 23-66. ISSN: 0938-8974, 1432-1467. DOI: 10.1007/s00332-019-09567-y. 
${ }_{1038}$ 75. LigPrep. New York, NY, 2019. articles/PMC4385651/. org/document/4160265/. System, Version 1.8”. 2015. inkscape. org/. $1007 / \mathrm{s} 10822-012-9584-8$
66. Renata De Paris et al. "Clustering Molecular Dynam- 104577 ics Trajectories for Optimizing Docking Experiments". ${ }_{1046}$ In: Computational Intelligence and Neuroscience 20151047 (2015). ISSN: 1687-5265. DOI: 10.1155/2015/916240. 1048 URL: https : / / www . ncbi . nlm . nih . gov / pmc / 1049

67. Travis E Oliphant. Guide to NumPy. Austin, Tex.: Con- ${ }^{1051}$ tinuum Press, 2015. IsBn: 978-1-5173-0007-4.

68. Robert T. McGibbon et al. "MDTraj: A Modern Open ${ }^{1053}$ Library for the Analysis of Molecular Dynamics Trajec- ${ }^{1054}$ tories". In: Biophysical Journal 109.8 (2015), pp. 1528- ${ }^{1055}$ 1532. ISSN: 00063495. DoI: $10.1016 / \mathrm{j} . \mathrm{bpj} .2015 .1056$ Q8. Q15. uRL: https: / / linkinghub. elsevier. com/ ${ }^{1057}$ retrieve/pii/S0006349515008267.

69. John D. Hunter. "Matplotlib: A 2D Graphics Environ- ${ }^{1059}$ ment". In: Computing in Science \& Engineering $9.3{ }^{1060}$ (2007), pp. 90-95. ISSN: 1521-9615. DOI: 10.1109/ 1061 MCSE . 2007 . 55. URL: http : / / ieeexplore , ieee . 1062

70. Schrödinger, LLC. "The PyMOL Molecular Graphics 1064

71. Thomas A. Halgren et al. "Glide: A New Approach for ${ }^{1066}$ Rapid, Accurate Docking and Scoring. 2. Enrichment ${ }^{1067}$ Factors in Database Screening". In: Journal of Medicinal ${ }^{1068}$ Chemistry 47.7 (2004), pp. 1750-1759. IssN: 0022-2623, ${ }^{1069}$ 1520-4804. DOI: $10.1021 /$ jm030644s. URL: https : ${ }^{1070}$ //pubs.acs.org/doi/10.1021/jm030644s.

72. B. et al Harrington. Inkscape. 2004. URL: http: //WwW. ${ }^{1072}$

73. Paul C. D. Hawkins et al. "Conformer Generation with OMEGA: Algorithm and Validation Using High Quality Structures from the Protein Databank and Cambridge Structural Database". In: Journal of Chemical Information and Modeling 50.4 (2010), pp. 572-584. IsSN: 1549-9596, 1549-960X. DOI: 10.1021/ci100031x. URL: https://pubs.acs.org/doi/10.1021/ci100031x.

74. Mark McGann. "FRED and HYBRID docking performance on standardized datasets". In: Journal of ComputerAided Molecular Design 26.8 (2012), pp. 897-906. ISSN: 0920-654X, 1573-4951. Dor: 10.1007/s10822-012$9584-8$. URL: http : / / link . springer . com / 10 . ${ }_{1086}$

6. Richard A. Friesner et al. "Extra Precision Glide: Dock- 1089 ing and Scoring Incorporating a Model of Hydrophobic 1090 Enclosure for Protein-Ligand Complexes". In: Journal of 1091 Medicinal Chemistry 49.21 (2006), pp. 6177-6196. ISSN: 1092 0022-2623, 1520-4804. DOI: 10 . 1021/jm051256o. URL: 1093 https://pubs.acs.org/doi/10.1021/jm051256o.
77. Bing Xie, John D. Clark, and David D. L. Minh. "Efficiency of Stratification for Ensemble Docking Using Reduced Ensembles". In: Journal of Chemical Information and Modeling 58.9 (2018), pp. 1915-1925. ISSN: 15499596, 1549-960X. DoI: 10.1021/acs. jcim. 8b00314. URL: https: / / pubs . acs . org/doi/10.1021/acs . jcim. 8b00314.

78. SciPy 1.0 Contributors et al. "SciPy 1.0: fundamental algorithms for scientific computing in Python". In: Nat Methods 17.3 (2020), pp. 261-272. ISSN: 1548-7091, 1548-7105. DoI: 10.1038/s41592-019-0686-2. URL: http: //www . nature. com/articles/s41592-019Q686-2.

79. Humphrey, W., Dalke, A. and Schulten, K. "VMD - Visual Molecular Dynamics". In: 14 (1996), pp. 33-38. URL: http: //www. ks . uiuc. edu/Research/vmd/.

80. John Stone. "An Efficient Library for Parallel Ray Tracing and Animation". MA thesis. Computer Science Department, University of Missouri-Rolla, 1998.

1. Zhiyong Zhou et al. "Comparative Performance of Several Flexible Docking Programs and Scoring Functions: Enrichment Studies for a Diverse Set of Pharmaceutically Relevant Targets". In: Journal of Chemical Information and Modeling 47.4 (2007), pp. 1599-1608. IsSN: 1549-9596, 1549-960X. DOI: 10.1021/ci7000346. URL: https://pubs.acs.org/doi/10.1021/ci7000346.

1071 82. Ward, Y.D., Emmanuel, M.J., Thomson, D.S., Liu, W., Bekkali, Y., Frye, L.L., Girardot, M., Morwick, T., Young, E.R.R., Zindell, R., Hrapchak, M., DeTuri, M., White, A., Crane, K.M., White, D.M., Wang, Y., Hao, M.-H., Grygon, C.A., Labadia, M.E., Wildeson, J., Freeman, D., Nelson, R., Capolino, A., Peterson, J.D., Raymond, E.L., Brown, M.L., Spero, D.M. Cathepsin S complexed with Compound 26. 2007. Dor: http://doi . org/10.2210/ $\mathrm{pdb} 2 \mathrm{R} 9 \mathrm{~N} / \mathrm{pdb}$.

83. Eddy Elisée et al. "Performance evaluation of molecular docking and free energy calculations protocols using the D3R Grand Challenge 4 dataset". In: Journal of Computer-Aided Molecular Design 33.12 (2019), pp. 1031-1043. ISSN: 0920-654X, 1573-4951. DOI: 10. 1007 / s10822-019-00232 - w. URL: http : / / link. springer.com/10.1007/s10822-019-00232-w.

84. Zeynep Kurkcuoglu et al. "Performance of HADDOCK and a simple contact-based proteinligand binding affinity predictor in the D3R Grand Challenge 2". In: Journal of Computer-Aided Molecular Design 32.1 (2018), pp. 175-185. ISSN: 0920-654X, 1573-4951. DOI: 10 . 1007 / s10822 - 017-0049-y. uRL: http : / / link. springer.com/10.1007/s10822-017-0049-y. 
1094 85. Susan L. McGovern and Brian K. Shoichet. "Information 1095 Decay in Molecular Docking Screens against Holo, Apo, 1096 and Modeled Conformations of Enzymes". In: Journal of $1097 \quad$ Medicinal Chemistry 46.14 (2003), pp. 2895-2907. IsSN: 1098 0022-2623, 1520-4804. DOI: 10.1021/jm0300330. URL: 1099 https://pubs.acs.org/doi/10.1021/jm0300330.

100 86. Gerhard Klebe. "Virtual ligand screening: strategies, $1101 \quad$ perspectives and limitations". In: Drug Discovery Today ${ }_{1102} 11.13$ (2006), pp. 580-594. ISSN: 1359-6446. DOI: 10. ${ }_{1103} 1016 / \mathrm{j}$.drudis .2006.05.012. uRL: https: / / wWW . 1104 ncbi.nlm.nih.gov/pmc/articles/PMC7108249/.

1105 87. Yuwei Yang et al. "Exploring fragment-based target1106 specific ranking protocol with machine learning on cathep1107 sin S". In: Journal of Computer-Aided Molecular Design $1108 \quad 33.12$ (2019), pp. 1095-1105. ISSN: 0920-654X, 1573${ }_{1109}$ 4951. DOI: $10 \cdot 1007$ / S10822-019-00247-3. URL: 1110 http : //link. springer. com/10.1007/s108221111 019-00247-3. 